KCP-613-5244

Distribution Category UC-706

\title{
QUALITY METRICS FOR PRODUCT DEFECTIVENESS AT KCD
}

\section{J. V. Grice}

Published July 1993

KCD Quality Metrics Committee:

M. L. Busby

R. G. Gemeinhardt

J. V. Grice

J. W. Hilton

D. R. Lemon

D. C. Wiercinski

L. J. Wright 


\section{Contents}

$\begin{array}{lll}\text { Section } & \text { Page }\end{array}$

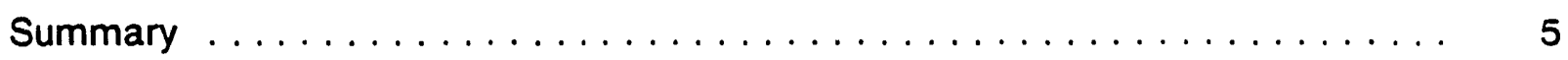

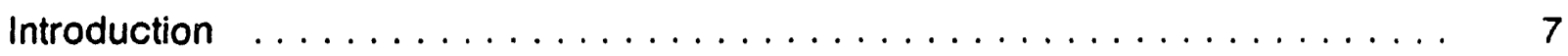

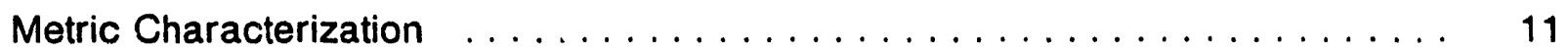

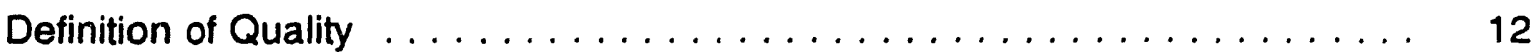

Common Features of Metrics $\ldots \ldots \ldots \ldots \ldots \ldots \ldots \ldots \ldots \ldots$

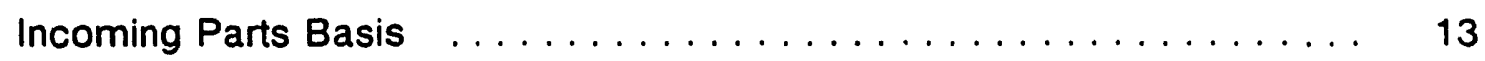

Defect Severity Weighting $\ldots \ldots \ldots \ldots \ldots \ldots \ldots$

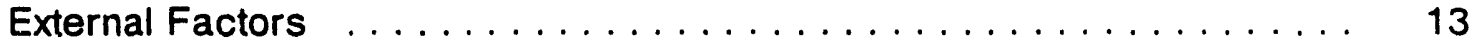

Factors That Affect Metrics $\ldots \ldots \ldots \ldots \ldots \ldots \ldots \ldots \ldots \ldots \ldots$

Samples Versus Parts $\ldots \ldots \ldots \ldots \ldots \ldots \ldots \ldots \ldots$

Sampling Plans $\ldots \ldots \ldots \ldots \ldots \ldots \ldots \ldots \ldots \ldots$

Counting Observations $\ldots \ldots \ldots \ldots \ldots \ldots \ldots \ldots \ldots$

KCD Metrics $\ldots \ldots \ldots \ldots \ldots \ldots \ldots \ldots \ldots \ldots \ldots \ldots \ldots \ldots \ldots \ldots$

Descriptions of Metrics $\ldots \ldots \ldots \ldots \ldots \ldots \ldots \ldots \ldots \ldots$

Percent Accepted Trouble Free $\ldots \ldots \ldots \ldots \ldots \ldots \ldots$

Percent Parts Accepted $\ldots \ldots \ldots \ldots \ldots \ldots \ldots \ldots \ldots$

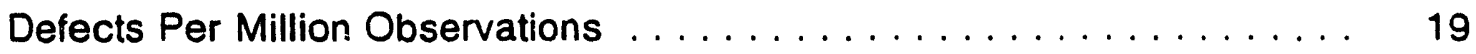

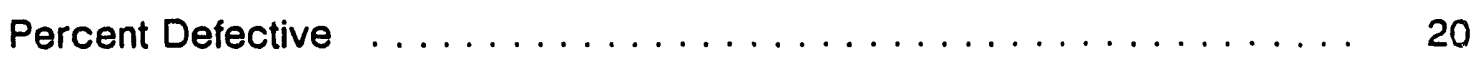

Purchased Material Applications $\ldots \ldots \ldots \ldots \ldots \ldots \ldots \ldots \ldots \ldots$ 


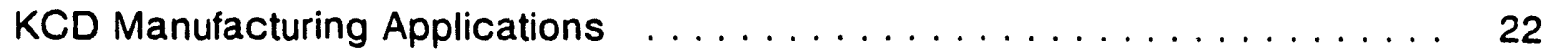

Use of Metrics as Management Tools $\ldots \ldots \ldots \ldots \ldots$

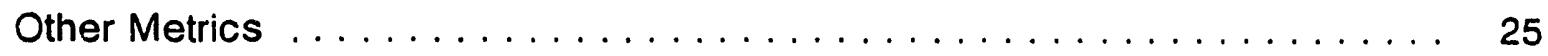

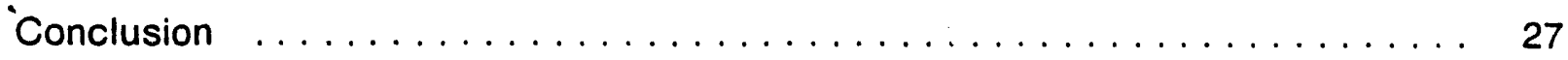

Appendices

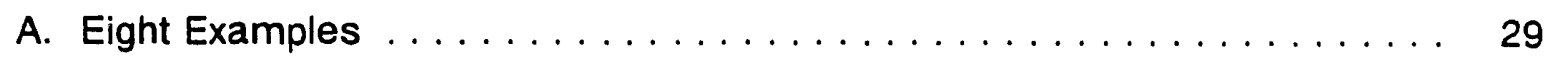

B. DPMO for an Electronics Assembly $\ldots \ldots \ldots \ldots \ldots \ldots$

C. DPMO Example in Mechanical Purchased Products $\ldots \ldots \ldots \ldots \ldots$ 


\section{Summary}

Metrics are discussed for measuring and tracking product defectiveness at AlliedSignal Inc., Kansas City Division (KCD). Three new metrics, the metric (percent defective) that preceded the new metrics, and several alternatives are described. The new metrics, Percent Parts Accepted, Percent Parts Accepted Trouble Free, and Defects Per Million Observations, (denoted by PPA, PATF, and DPMO, respectively) were implemented for KCD-manufactured product and purchased material in November 1992. These metrics replace the percent defective metric that had been used for several years. The PPA and PATF metrics primarily measure quality performance while DPMO measures the effects of continuous improvement activities. The new metrics measure product quality in terms of product defectiveness observed only during the inspection process. The metrics were originally developed for purchased product and were adapted to manufactured product to provide a consistent set of metrics plant-wide.

The new metrics provide a meaningful tool to measure the quantity of product defectiveness in terms of the customer's requirements and expectations for quality. Many valid metrics are available and all will have deficiencies. These three metrics are among the least sensitive to problems and are easily understood. They will serve as good management tools for $K C D$ in the foreseeable future until new flexible data systems and reporting procedures can be implemented that can provide more detailed and accurate metric computations. 


\section{Introduction}

One of the primary objectives of a quality management system is to measure and track product quality. Selection of proper metrics supports effective management and decision support. The objectives of the metrics for manufactured and purchased products and materials at AlliedSignal Inc., Kansas City Division (KCD), are to perform the following:

- Measure the quality of incoming material and, thereby,

- Measure the effectiveness of the generating systems.

Three metrics, Percent Parts Accepted, Percent Parts Accepted Trouble Free, and Defects Per Million Observations, are designated as the primary metrics for part defectiveness at KCD. The metric Percent Defective and its complement, Percent Accepted, were the primary metrics prior to the implementation of the current metrics in November 1992. These metrics and some brief characteristics are listed below.

- Percent Accepted Trouble Free (PATF)--PATF is the percent of parts submitted to inspection that was accepted without additional effort and cost beyond that projected in the inspection instructions. It measures the system's ability to meet customer expectations of providing product that is accepted at expected cost. This is product accepted with no trouble found but not necessarily with zero defects. This metric has also been known as "Percent Accepted Defect Free."

PATF is computed using the following equation.

$$
\text { PATF }=100 \times \frac{\text { Total Number of Parts Accepted Trouble Free }}{\text { Total Number of Parts Submitted }}
$$

- Percent Parts Accepted (PPA)--PPA is the percent of parts submitted to inspection that was accepted, regardless of the effort required to gain acceptance. It measures the system's ability to meet the customer's need to provide usable product.

PPA is computed using the following equation.

$$
P P A=100 \times \frac{\text { Total Number of Parts Accepted }}{\text { Total Number of Parts Submitted }}
$$

- Defects Per Million Observations (DPMO)--DPMO is the average number of inspection observations that was found defective per million of inspection observations inspected. It measures defectiveness on the basis of the proportion of characteristics observed defective within parts. It measures the effects of quality improvement activities on 
product defectiveness by measuring defectiveness on a smaller unit of resolution, the inspection observation of a part characteristic.

DPMO is computed using the following equation.

$$
\text { DPMO }=\frac{\text { Total Number of Defective Inspection Observations }}{\text { Total Number of Inspection Observations }} \times 1,000,000
$$

- Percent Defective (PD)--This metric was used prior to the implementation of the three new metrics. It is the percent of parts inspected that was found defective in a shipment or inspection submittal. PD was intended to measure the level of defectiveness in incoming product (either purchased or produced in house). It and its complement, Percent Accepted (PA = 100 - PD) measured the system's likelihood of meeting the customer expectation of providing conforming parts.

PD is computed using the following equation.

$$
P D=100 \times \frac{\text { Total Number of Parts Defective }}{\text { Total Number of Parts Inspected (Largest Sample Size) }}
$$

The totals are taken over the inspection operations (groups) for manufacturing and over the distinct shipments for purchased material. In the manufacturing areas, the number of defective parts at a group is the number of units found defective among the keys (characteristics) within the group. For purchased material, the number of defective units is the maximum number of units found defective among the keys. The number inspected is the maximum number inspected among the keys in the group for the submittal or shipment. In most cases, the two numbers of defectives will be the same but the sample size used may not be from the same key.

The metrics used to measure quality depend on the definition of quality that is being used. The current definition emphasizes meeting customer requirements and expectations. This definition of quality has several characteristics to measure. A single metric will not provide adequate tracking of all the desirable characteristics. Often metrics are valid for only a short period of time. As the system is improved, new metrics are required to measure the new level of quality achievement and subsequent improvements.

The primary problem with all metrics devised for this type of application is that the metrics attempt to measure a quantity across a heterogeneous mix of products that continually changes over time. This means that the data system that collects and manipulates the data is a critical feature of the system that uses metrics. Some of these issues ivill be illustrated. The QIM/Trace project in the Focused Factory and the CACTUS system have the potential to better collect data and calculate the metrics.

Three new metrics plus the old percent defective metric will be discussed. Although the metrics are designed to measure the quality of incoming or submitted product, they also support the measurement of system effectiveness by facilitating comparisons such as 
before versus after corrective action and among suppliers. Each has merit as well as drawbacks for specific aspects of the system that is measured.

The first section discusses the characterization of the metrics. The next section describes the metrics and their application to KCD-purchased and manufactured product. The appendices include several examples of sampling and counting results. These examples illustrate some of the effects and anomalies that may be present in the computation of the metrics.

Another critical feature is the manner by which the counting of defects and parts and the metric computations are performed. The appendices illustrate some examples. 


\section{Metric Characterization}

The discussion on quality metrics begins by first analyzing the criteria that, in general, characterize metrics. Then the discussion will focus on issues that involve measuring quality in terms of product defectiveness. In particular, metrics that measure quality at KCD share some common features and are affected by some common factors.

A metric is a numerical representation of the state of the system that is of interest. A metric is typically characterized by four fundamental criteria.

- The state of the system of interest is a quantity.

- A metric defines a complete ordering of the states of the system (that is, quantities) being measured.

- The ordering must be nondegenerate.

- A metric transforms the ordering to a meaningful scale.

The state of interest is a quantity means that the state (or condition) is present in the system in varying degrees of magnitude. A simple example is the size of a physical object as represented by the length. The complete ordering means that one can determine the larger (and smaller) of any possible pair of system states or declare the pair equal. Nondegenerate means that at least one state is measured larger than one other state; that is, not all states are declared equal. The scale provides the numerical value that corresponds to the system state. Many physical conditions are quantities, such as density, velocity, volume, and temperature. That fact that the usual metrics for these examples meet these criteria is easily seen. The length example is the primary intuitive model for other less obvious metrics.

The task for developing a metric to measure product defectiveness is to carefully define the quantity in the context of the first three criteria, then identify a numerical scale that is meaningful in a commonsense and practical way. For example, consider the number (quantity) of people in the plant that are working in inspection. The ratio of the inspection people to the total meets the first three criteria. If one scales this quantity in terms of the percentage of people in inspection, the resulting numbers provide a meaningful basis for comparison. Another example is to scale in terms of a count per unit of area or time such as the count of the number of events during the course of a year or other unit of time.

An additional aspect of the nature of a meaningful scale is the desire that the scale should be finite on at least one end of the scale and the end point on that end represents perfection. That is, when absolutely no defectiveness is present, the value on the scale should be the finite end point. Optionally, when the total population is defective, a second end point may be specified that represents this extremity. For example, consider an urn 
of red and white beads. One may choose the quantity to be the degree of red beads in the urn and the proportion of red beads in the total to be the numeric scale. The proportion scale is limited to the interval from zero to one. At zero, the urn contains only white beads (no red beads) and at one, only red beads (no white beads). The other criteria may be easily verified.

\section{Definition of Quality}

The definition of product quality applicable at KCD is the definition of quality from DOE $A L$ Quality Criteria (QC-1), Rev. 7. It states three characteristics of quality.

- Quality is conformance to customer requirements and expectations.

- Quality is enhanced through a process of continuous improvement which focuses on the prevention of errors and reduction in variability in processes, products, and services.

- A disciplined quality system, employing costs of nonconformance to assess its effectiveness, will be used and should yield reduced operating costs and increased productivity.

This definition of quality has three quantities that are of interest that correspond to the metrics under consideration. The degree of conformance to requiremerits and expectations are separated into two quantities, and enhancement to quality through continuous improvement constitutes the third. These three quantities are different from the quantity that was scaled by the "percent defective" metric previously used at KCD. This metric was intended to reflect the degree of conformance to customer requirements and expectations by scaling the values in terms of the complement, the degree of nonconformance. Although the concepts are complementary, the underlying quantities are not equivalent.

\section{Common Features of Metrics}

The four metrics considered in this discussion, PATF, PPA, DPMO, and PD, have some common features while measuring different quantities in the quality system. The common features focus on issues in measuring quality of incoming parts and treating all defects as having equal severity.

The three common features are as follows.

- All seek to measure the quality of parts coming into the system and not of parts that are sent on to stores or next assembly.

- None of the metrics consider the relative severity of defectiveness, such as rating defects as major, minor, or incidental, except as a result of variation in sample sizes for different characteristics. 
- All are affected by external factors such as changes in shipment sizes, sample sizes, the way bulk material is combined with discrete parts, and the number of and manner by which characteristics are identified.

These features are described below.

\section{Incoming Parts Basis}

All four metrics attempt to measure the incoming product quality from the point of view of KCD as the buyer of the product, not the user of the product. None of the metrics estimate the defectiveness rate in outgoing parts. Inspection plays a significant role in removing defective parts before sending parts to stores. The rate of defectiveness in the outgoing parts should be no higher and can be significantly lower than that of the incoming parts.

To measure the outgoing quality level would be difficult. KCD currently collects little, if any, data that tries to directly assess the defectiveness of product in stores or next assembly. Failure analysis is performed on defective product, but the results are not integrated into the overall quality data system and, in particular, are not used in setting supplier ratings or assessing production performance.

\section{Defect Severity Weighting}

None of the metrics directly consider the criticality or severity of defects. A functional failure is counted the same as a cosmetic paint scratch, assuming both are listed in the requirements. KCD generally uses the sampling plan as an indirect means to weight the severity of defects. The sampling plan is selected to provide relative limits of risk of defects according to the characteristic's criticality. Characteristics that have a $100 \%$ inspection callout are more critical than characteristics with a 5.0 LTPD sampling plan (for example, $n=77, c=1)$. A characteristic with a standard $(n=15, c=0)$ callout is even less critical. Thus, the sample size can limit the number of defects that are counted in the metric. Example $A$ in Appendix A illustrates this situation.

\section{External Factors}

All four metrics can be affected by changes in inspection rates (for example, change from $100 \%$ to acceptance sampling or vice versa). However, all will converge to the desired target (usually 100 or zero percent) when part defectiveness goes to zero. For example, if a $100 \%$ callout is reduced to sampling at $(n, c)=(45,0)$, for example, then shipments that contain defective parts may be accepted that would contribute defectives under $100 \%$

inspection. The actual effect depends on the shipment size and defectiveness rates. See Appendix A for examples of the effects of shipment size and sample size. 


\section{Factors That Affect Metrics}

Several factors can affect the values assigned by the metrics. These factors will cause different metric values to be assigned to the same conditions. These variations don't invalidate the metrics, but, to fully understand the values assigned, the possible underlying effects must also be understood. Four of these effects are described below. Values assigned by the metrics may be due to one or more of the following:

- the choice of the number of samples (parts inspected) or the number of received parts (inspected or not) as the basis of the metric,

- the choice of different sampling plans for characteristics within a part, and

- the manner by which characteristics (inspection observations) are identified and counted on parts.

\section{Samples Versus Parts}

In the case of samples versus parts received, the metrics are measuring different quantities even though there is a strong correlation between the two. When the denominator, that is, the basis, for the metric is the number of entities inspected, for example, shipments, parts, or observations, the metric is attempting to measure the average rate of defective entities in the population generated by the processes. When the basis is the total entities received, the metric is attempting to measure the rate at which entities are rejected (or accepted). In practice, thes? rates can be and are different, but both rates will tend to their desirable targets as the leval of defectiveness decrease:s. Situations can occur where variation in the basis or the inspection results will be reflected in some metrics while not reflected in the others. See the appendices for examples of these effects.

\section{Sampling Plans}

When different sampling plans are applied to distinct characteristics on a part, the effect is a weighting of the severity of defects by the sample size. This weighting is based on the fact that as sample size increases so does the number of defectives found for a given defect rate. These effects are evaluated in the discussion of the specific metrics that follows. The appendices contain examples.

\section{Counting Observations}

The decomposition of the part into the part characteristics to be inspected dictates the types and number of defects that are sought. This process is driven by the part requirements, but the actual result may be more or less complex, depending on the responsible engineer's judgment and approach to the task. Generally, less complex inspection tends to find fewer defects than more complex inspection. More defects usually mean more defective parts. The structure and basis of the metrics may or may 
not be responsive to these changes. In particular, the metric DPMO is sensitive to this situation. The discussion in the next section on DPMO will review this issue in more detail. 


\section{KCD Metrics}

Four metrics will be discussed in detail. The first three are the new metrics currently implemented for reporting defectiveness for both purchased material and manufacturing, PATF, PPA, and DPMO. The last, PD, is the predecessor to the current three. The metrics will ive described, including a description of the methods to compute the metrics, issues that impact the computations, and the quantity that the metric measures. Applications to purchased material and manufacturing will be compared and contrasted, and general usage of the metrics as management tools will be discussed. Several other metrics are listed for completeness. Some of these metrics are in use in a variety of places or have been used at KCD in the past.

\section{Descriptions of Metrics}

\section{Percent Accepted Trouble Free}

The first new metric considered is Percent Accepted Trouble Free (PATF). PATF is the proportion of parts received that was accepted without any further effort at the Kansas City Plant (KCP) beyond the prescribed inspection and handling (that is, submittals pass all prescribed sampling and testing requirements in inspection). The basis for this metric is the number of parts accepted on the first try, whether or not they were inspected, versus the total parts received.

PATF is computed using the following equation.

$$
\text { PATF }=100 \times \frac{\text { Total Number of Parts Accepted Trouble Free }}{\text { Total Number of Parts Submitted }}
$$

PATF measures the efficiency (or ability) of the system to meet customer expectations for conforming submittals or shipments of product at agreed cost. In this case, conforming is assumed to mean acceptable. It includes only parts that were shipped to stores (or were equivalent, such as D-test units that passed) with no trouble found. It does not include parts screened for failing sampling inspection and does not include parts that have been scrapped, reworked, or identified in Specification Exceptinn Reiease (SXR), Advance Change Order (ACO), or Return to Vendor (RTV) documentation.

The totals are counted across all first-time submittals (PM01 for KCD manufacturing areas) made during the designated time period. This method makes for a simple metric to compute. However, this does not make any allowance for the differences among dissim ilar product. the relative quantities suibmitted per product or inspection group, or the 
sample sizes specified in the inspection instruction. If these characteristics change, the metric's value may change when no real change occurred in the defectiveness.

PATF will react more consistently to changes to incoming quality than will PPA but less consistently than DPMO and should react about the same as PD. For example, suppose a chronic defect is corrected in a product. Defect-iree acceptance is now dependent on the absence of other defects. If no othei defects are present, PATF will reflect the improvement (as will PD). If other defects are still present, PATF (and PD) will only reflect the incremental improvement beyond the remaining defects.

PATF is less sensitive to changes in sample size than PD because it depends on the number of parts submitted to inspection and their disposition and less on the actual sample size. Sensitivity, in this case, means the relative size of changes that can be easily detected. It is more sensitive to rejection of the submittal by sampling because when a shipment is rejected by the sample, none of the parts in the remainder shipment are counted as accepted, even though the sample may have contained only one defective. See the appendices for examples.

\section{Percent Parts Accepted}

The second of the new metrics to consider is Percent Parts Accepted (PPA). PPA is the proportion of parts received that was accepted for use. The reason for acceptance is of no concern. Acceptance of a submittal of parts may be on a sampling basis or, if defects are found, acceptance may be achieved by SXR, ACO, screening, or rework. The basis for this metric is the number of parts accepted versus the number submitted.

PPA is computed using the following equation.

$$
\text { PPA }=100 \times \frac{\text { Total Number of Parts Accepted }}{\text { Total Number of Parts Submitted }}
$$

PPA measures the efficiency (or ability) of the system (suppliers and KCD) to meet the customer's needs; that is, "Can usable submittals of product be acquired, manufactured, and delivered?" Again, conforming means acceptable, but acceptance may be judged relative to the original requirements, to some approved variation of the requirements (for example, SXR, ACO), or due to extra effort by KCD (for example, screen or rework). The disposition of the parts is the important feature rather than the observed defectiveness.

PPA reflects the parts which were actually purchased or credited to the production organization and does not include parts scrapped or, for purchased material, returned to the vendor/supplier (RTV). It should include any acceptable D-test parts, parts removed for engineering evaluation, parts accepted by SXR or ACO, and parts that were screened or reworked and later accepted.

PPA is the least sensitive of the four metrics in detecting changes in the overall defectiveness level. For exa.nple, suppose product is accepted by SXR for marking 
defects and the supplier implements corrective action to ensure properly marked parts in future shipments. The SXR'd product is accepted just as the future shipments with the corrert marking will be accepted. The PPA value will not reflect any change after the corrective action has been implemented. If the product had been scrapped or returned to the supplier instead of being SXR'd, PPA would have reflected the change.

The difference from PATF to PPA is the percentage of product that was acceptable but required additional effort and cost to ultimately accept. This represents, in an indirect way, a cost of nonconformance in that if all product had been acceptable, the additional cost would not have been expended. The difference from PPA to 100 percent represents the units that could not be accepted for any reason. This represents a different type of cost of nonconformance. Monitoring these differences is a means of validating direct measures of cost of nonconformance.

\section{Defects Per Million Observations}

The third metric is Defects Per Million Observations (DPMO). DPMO is the proportion of inspections observations that were found defective. This metric uses a finer unit of resolution than the other metrics. This unit of resolution is the inspection observation, that is, a chance for the inspection process to find a defect. A more formal definition for inspection observation is offered below. The basis for this metric is the number of inspection observations scaled to a rate per one million $(1,000,000)$ inspection observations. For example, if 200 characteristics were found defective in 10,000 inspections observations, the proportion of 0.02 is computed. Then the proportion of 0.02 is reported as $0.02^{\star} 1,000,000$ or 20,000 Defects Per Million Observations. This scaling is no different than scaling to a percentage (that is, per 100) except the values will still be whole numbers when defectiveness is quite low.

DPMO is computed using the following equation.

$$
D P M O=\frac{\text { Total Number of Defective Inspection Observations }}{\text { Total Number of Inspection Observations }} \times 1,000,000
$$

An inspection observation is defined as a feature or characteristic that is inspected on the part. The quality engineer identifies the characteristics to be inspected in the inspection instructions. The number of observations varies from product to product. Simple parts will have only a few observations while complex parts will have many observations. This change of resolution from parts to observations is similar to changing from shipments to parts. Because the resolution is smaller, smaller changes can be detected. This also serves to weight parts by part complexity to the extent that the number of characteristics represents the complexity. The total in the denominator represents the inspection observations that were actually inspected.

The way DPMO is computed is critical to its interpretation. It is based on the inspection observation, however that is identified. The next logical step would be to use a metric that is based on the production opportunity to create a defect. This level of resolution is 
desirable as process characterization, control, and capability become more widely and uniformly applied. This would allow the metric to truly reflect the contribution of processes to the overall level of defectiveness and provide the means to trace defectiveness to the generating process.

DPMO more readily detects the incremental effects of supplier and KCD continuous improvement activities than does PPA or PATF. For example, when the root cause of a defect is identified and removed from the system, the defects do not appear although the observations are still counted. The computation will reflect this change even if other defect types are still present.

\section{Percent Defective}

The metric previously used by KCD is Percent Defective (PD). PD is computed as the ratio of the largest number of defectives observed on a characteristic to the largest sample size inspected in the part submittal. PD estimates the overall rate at which supplier's parts are defective and is the complement to the calculation that has been called Percent Accepted.

$P D$ is computed using the following equation.

$$
P D=100 \times \frac{\text { Total Number of Parts Defective }}{\text { Total Number of Parts Inspected (Largest Sa mple Size) }}
$$

The totals are taken over the inspection operations (groups) for manufacturing and over the distinct shipments for purchased material. In the manufacturing areas, the number of defective parts in a group is the number of unique units found defective among the keys (characteristics) within the group. For purchased material, the number of defective units is the maximum number of units found defective among the keys. The number inspected is the largest sample size inspected among the keys in the group of KCD-manufactured product or the Inspection Instruction (II) for the shipment. In most cases, the two numbers of defectives will be the same but the sample size used may not be from the same key.

This metric weights the contribution to the overall rating according to the sample size taken at inspection. The basis is the number of parts inspected, not parts received. Thus, it is sensitive to variations in sample size. It can be artificially low if some part characteristics have high defectiveness rates, sampling and $100 \%$ callouts, and large shipment sizes. See Examples $A$ and $E$ in Appendix $A$.

It can be artificially high if parts have small sample size callouts and low to moderate defectiveness. Acceptance sampling plans with small sample sizes typically have an acceptance number of zero $(c=0)$. These plans tend to accept small to moderate defectiveness and in the process find no defectives to add to the numerator. See Example $D$ in Appendix $A$. 


\section{Purchased Material Applications}

The Supplier Quality Evaluation System (SQES) is a quality information system intended to track the performance of KCD's suppliers. These suppliers provide bulk material, pieceparts, and assemblies for production activities at KCD. The three new metrics provide effective support for the objectives for purchased material quality.

The primary objectives of the metrics are to measure and track supplier product quality. These metrics measure the ability of the purchased product system to acquire conforming product. These metrics can be inappropriately affected by the improper inclusion of the results from bulk material. The metrics will be more representative of the changes in the defectiveness rate if the bulk material component is reported separately or included on the basis of shipments or possibly of shipping container (for example, spools of wire or drums of solvent).

Conforming refers to the shipment level for purchased material, not the individual part level, in this discussion. A shipment that meets its acceptance criteria as specified in the inspection instructions is considered conforming even though some characteristics were accepted on a sampling inspection basis. An accepted shipment may contain nonconforming parts not identified by the specified sampling plan. The frequency of nonconforming parts and types of nonconformances will be consistent with the risks allowed by the sampling plan.

By the nature of the products, the measurement of quality of bulk materials and the measurement of quality of discrete parts are inconsistent. This is due to the fact that the basic unit for counting purposes within a shipment is different. A shipment of discrete parts has a physically well-defined basic unit in the shipment. A shipment of bulk material does not have a basic physical unit for counting. The material is purchased on the basis of a convenient unit of measure, such as feet, tons, or gallons. This material is usually continuous or connected and the units are easily scaled to other units of measure. These inconsistencies cannot be easily reconciled because the choice of units is arbitrary. Thus, the measurement of quality for the two areas should be separate unless consistent counting units can be identified.

For bulk materials, one appropriate counting basis is the shipment. Shipments are often consistent in interpretation and disposition. This is due to the discrete nature of shipments. Testing requirements are assumed to apply to the whole of the shipment. Inspection is usually by sampling, and acceptance and disposition apply to the whole shipment. Another possible basis is the shipping container, such as a bucket of nails, a spool of wire, or a 55-gallon drum of solvent. Shipping containers of bulk material typically cannot be split or screened. The percent of shipments or shipping containers accepted would be valid metrics for bulk material in the context of combining the result with the results from discrete parts. 


\section{KCD Manufacturing Applications}

The three current metrics are applicable to KCD manufacturing areas in the same context as for purchased material. There are some slight differences in their interpretation and the quantities that they measure. This is due to different objectives in measuring quality in the factory areas and different data systems for collecting and storing inspection results data.

The objectives in measuring quality in the factory area are

- To measure the ability of the factory to produce and deliver parts to an inspection point that conforms to their requirements and

- To measure the effects of continuous improvements activities.

These objectives are somewhat different than those in the purchased product area but share some common areas. Application of the three metrics, PPA, PATF, and DPMO, in either purchased material or the manufacturing areas is a similar task. The miatrics are measuring the same quantities with only small differences. As in purchased material, the metrics computations try to exploit existing data systems. In purchased material, the Supplier Quality Evaluation System (SQES) is used. In manufacturing, the Quality Attributes Reporting System (QARS) is used.

Two competing issues that affect DPMO and are not representative of changes in defectiveness are the identification of the inspection characteristics and the counting of the observations. The first is that the more characteristics that are identified on the Inspection Instruction (II) and inspected, the higher the number of observations. The second is that each characteristic identified may propagate multiple observations.

In the KCD manufacturing area, product has value added by forming, fabricating, or assembling pieceparts into higher level products. At several points in this process the parts are sent to inspection, and the value added is credited by the accounting division. This is the basis of what is referred to as "parts" in discussing the metrics for the manufacturing areas, even though many more operations may be added to the parts. Note that each manufacturing process transforms the parts into different parts according to the value added. Some parts may pass through several inspections and be counted in the metrics at each inspection point. When this happens, the inspections often focus on different areas of characteristics, for example, visual and electrical performance. Thus, each counting is generally based on an appropriate portion of the new value added. This phenomenon was deemed appropriate and allowed use of the existing data system, QARS, to perform the calculations on a routine basis. The QIM/Trace (Quality Information Management and product TRACEability) project in the Focused Factory initiative and other ancillary projects are developing the data systems of the future with these requirements in mind to ensure that the current or better capability is maintained. In particular, the Computer-Aided Collection, Tracking, and Updating System (CACTUS) collects data on the product disposition basis. 
In using QARS to supply the data and computations for the metrics, several issues arise. These issues involve the way in which defectives, inspection observations, and parts are counted. The issues relate to the manner by which sampling results are combined; when and how disposition, especially of defective product, is accomplished; and how inspection observations (part characteristics) are identified and counted.

The old PD metric was particularly sensitive to the way sampling results were reported and tallied. When using the new DPMO metric, these problems are reduced, because all the sample results are used and not just the largest number defective and largest number inspected in the inspection group. Sampling plan sample sizes and the number of characteristics identified now have an effect. Because sampling plans are used to manage risk on the characteristic sampled, the effect is arguably proper according to the perceived risk. Counting characteristics is not so clear an argument.

The number of characteristics identified and whether multiple inspection observations are counted on a characteristic identified in the inspection instruction have a predictable effect on DPMO. If the number of inspection observations increases and the number of defectives found remains constant, obviously, DPMO will be smaller. However, if the number of defects found goes up with the increase in observations, DPMO typically will stay the same and may go up. An example of these effects is presented in Appendix $A$.

The PPA and PATF metrics rely on the final disposition of product. When disposition is not timely and QARS is used, the values of PPA and PATF are affected. QARS reports results according to the date product is inspected, even though the disposition of the product may not be complete for rejected parts. The computation of PATF from QARS assumes that rejected product is not acceptable and the value is computed accordingly. PPA is computed based on all product found acceptable during the time period, regardless of whether or not it was first submitted for inspection during the time period. PATF is less affected than PPA by this situation. Other systems like CACTUS and QIM/Trace will have product disposition information than can be used in these metric computations.

\section{Use of Metrics as Management Tools}

The metrics are effective management tools. Their usage changes as the level of homogeneity increases from the top level global computation for the whole plant down through the business unit, department, and commodity group to the individual product. The metrics provide consistent and meaningful measures of the performance on the subject quantity. The current data systems can provide limited flexibility in tracing defectiveness to its source, but new data systems in development appear to offer better tools.

At the top or plant-wide level, the metrics provide the system-wide perspective. They can point to system-wide effects as reflected in changes in their values. These changes can then be compared to the historical variations using the usual data analysis techniques to assess whether the change was within the expected range or if it was beyond. If the values are then computed according to business unit, the same types of comparisons can 
be made at that lower level. In particular, if a value is out of expected range at the top level, it is likely that only one of the business units contributed the defectiveness that made the value undesirable.

The computation at the business unit level would make the identification easy or indicate that further investigation is proper. The computations could be pursued until the lowest level source of defectiveness is identified. This level may be the inspection of a single product group, a family of like parts, a department, the business unit, or the top level. This analysis gives management the perspective of the scope of effort required to reduce the defectiveness and the amount that is involved. As the isolation of the source of special defectiveness proceeds to a lower level, the corrective action required becomes more localized.

The comparison of PPA and PATF provides insight into the nature of the cost of nonconformance. As noted earlier, the amount that PPA falls short of 100 percent reflects the amount of nonconforming product that could not be accepted at all while the difference from PATF to PPA reflects the amount of nonconforming product that could be accepted with extra effort. The values can then be used to validate the cost of nonconformance data collected independently and identify the relative proportions of unusable to usablewith-help parts.

The DPMO metric is the best of the three in tracking continuous improvement activities. The effects of continuous improvement activities are reflected in the product defectiveness that is observed at inspection. As continuous improvement activities reduce defectiveness, the metric will become smaller. The effects will be more readily seen at the part, part family, or department levels, but system-wide effects should be visible in the overall metric.

The implementation of DPMO using the current QARS data system is somewhat limited. DPMO represents a compromise on this basis. Users of DPMO must recognize the limitations and understand how the metric is actually computed from QARS data.

In the current DPMO computation, inspection keys are counted as the inspection observations, but multiple-defect types can be reported for an individual key. The current structure in QARS does not allow the computations to be made on cross classifications of defect types, products, and departments. If the computations could be obtained for these combinations, the source of defectiveness could be better isolated to a smaller portion of the plant's production for the time period.

QARS would also be limited if a count of the inspection observations on a key were entered into an Inspection Instruction (II) database field and the reported defects used as the count of the defective observations. To expand the counting to allow multiple inspection observations on a single key, the II database and reporting to QARS would require some modifications.

A simple way to make this count is to embed the number of observations in the key description field. In this situation, the computation would be done by extracting the number of defects from the QARS results and the number of observations from the key 
description field. This method would work where the inspection is always going to perform all the predicted inspections and count all the defects found.

If automated inspection equipment is used and the inspection run on a part is aborted due to a defect, then the number of inspection observations may not accurately reflect the actual number of observations and the number found defective. These quantities would have to be counted and reported by the inspector. Much of the automated inspection equipment in the plant does not do this counting, and it would be expensive to make such modifications.

New data systems currently in development hold more potential to fully implement this kind of flexibility for the DPMO metric. One feature of the QIM/Trace system is that it would allow the QARS and the VAriables DAta system (VADA) to be merged. In this system, each part characteristic that is important to part quality is identified, whether its inspection is based on attributes or variables data, and the results are collected in a common database. A characteristic is identified according to whether it is worth collecting its results separately. The characteristics become the inspection observations. In this situation, many of the limitations discussed above will be resolved.

\section{Other Metrics}

Many meirics are available to measure product defectiveness. They measure quality of product from different points of view, have different bases of resolution, and exhibit varying. levels of consistency in reflecting changes in defectiveness.

Several of these metrics, their application (incoming or outgoing product), and their use, if any, outside KCD are described below. Most measure parameters similar to those described above and have similar limitations. All the following metrics are valid metrics under the definition and will converge to the desirable targets as the defectiveness rate decreases.

- Percent Lots Defective--AlliedSignal AiResearch uses this metric. It is computed monthly as the percent of lots rejected versus the lots received.

- Defects Per Unit (sampled units)--Some AT\&T divisions use this metric. It is computed as the number of defects observed in the inspected units divided by the number of inspected units. A more complicated version attempts to estimate the outgoing defect rate and uses a weighted average of the observed incoming and outgoing rates. Most of these applications employ the same sampling plan to all characteristics.

- Percent Defective (sampled units)--AlliedSignal AiResearch and Fluid Systems divisions use this metric. It is computed in the usual manner based on the number of defective parts in the total of the inspected units.

- Parts Per Million (PPM)--This is the same as percent defective except scaled to a rate in terms of defectives per million parts. A Honeywell division that is a KCD supplier and AlliedSignal Aerospace have been using this metric. 
- Cost Ratio--This ratio is computed as $100^{*}$ (cost of accepted parts)/(cost of parts received). It is included in the MRP-II software selected for KCD by the Focused Factory project and is recommended by the MRP-II system supplier. However, the supplier reports that it is seldom used. This metric requires extensive information on all costs associated with the procurement and processing of parts.

- Weighted Index--A numerical index based on weighted defect severity, that is, Major, Minor, and Incidental. This metric is often recommended in the quality sciences literature. There are several variations, but the central theme is to compute a weighted sum of defects per unit inspected. The relative contribution of an incidental defect will be only a fraction of that of a major defect. EG\&G Mound uses this approach. 


\section{Conclusion}

- The objectives of quality metrics are to measure quality according to the operational definition of quality and within the context of the quality system. As the system is improved, metrics will change in terms of what they measure and how well they measure it. At some point, new metrics will be needed. The proposed new metrics (PPA, PATF, and DPMO) are able to measure quality in a manner consistent with the QC-1 definition. PATF measures meeting customer expectations, PPA measures meeting customer requirements, and DPMO measures effects of continuous improvement efforts. All these metrics are subject to effects that are related to the task of providing a global value and meaningful scale when the results of dissimilar products must be combined to yield the value.

Measurements of product defectiveness may be made from the point of view of incoming product or outgoing product. Incoming is typically easier to measure because data is more readily available via inspection. To effectively measure outgoing defectiveness, defectiveness data must be readily available. In either case, the data must be integrated with other plant systems.

Many aspects of the inspection process can affect the estimates even though no real change in the rate has occurred. These aspects include changes in shipment sizes, changes in inspection sample sizes, types of defects, part configuration/characteristic similarities, changes in product mix, and changes in product and inspection definitions. Metrics typically do not attempt to cope with these factors. Most metrics view such factors as uncontrolled sources of variability and assume that, on average, the effects will not obscure the overall level and trends in the system. To measure the defectiveness level of outgoing parts, for example, parts shipped to stores or next assembly, new testing, inspection, and data collection activities are required. The data may come from defectives found and analyzed in two main areas, depending on the desired scope: next assembly inspection or audit samples and functional (that is, D-test/E-test or field) failures. 


\section{Appendix A}

\section{Eight Examples}


This appendix contains eight examples of shipments, inspection results, and metrics computations that illustrate many of the features of quality metrics for purchased product. Table A contains the eight examples. Appendices $B$ and $C$ follow with examples of the effects different observation counting strategies can have on the DPMO metric.

Each example consists of the inspection results from one of eight shipments of product. Three characteristics are inspected on the parts. The first, key X001, is inspected according to an ( $n=15, c=0$ ) sampling plan. The second, key $X 002$, is inspected according to an $(n=45, c=1)$ plan. The third, key $X 003$, is 100 percent inspected. Each key represents an opportunity for a defect on a part for DPMO computations.

Examples $A$ through $D$ are shipments of 50 pats each. Examples $E$ through $H$ are 1000 parts each. Examples $A$ and $E$ illustrate. defectiveness on a sampled key where the decision is to screen the shipment on that key. Examples $B$ and $F$ are similar except the shipment disposition is to return the shipment to the supplier (RTV). Examples $C$ and $G$ exhibit low to moderate defectiveness on sampled keys. Examples $D$ and $H$ have moderate defectiveness on multiple keys.

The computations were performed according to the following definitions. All defectives are assumed to be unique.

$$
\text { PATF }=100 \times \frac{\text { Total Number of Parts Accepted Trouble Free }}{\text { Total Number of Parts Sub mitted }}
$$

The Number of Parts Accepted Trouble Free is the line in the table that corresponds to the number of parts accepted without any additional. cost or effort.

$$
P P A=100 \times \frac{\text { Total Number of Parts Accepted }}{\text { Total Number of Parts Submitted/Received }}
$$

The Total Accepted and Total Received are the lines under Shipment Total corresponding to the total number of parts accepted (that is, not scrapped or not RT'V'd) and the total number of parts received.

$$
\text { DPMO }=\frac{\text { Total Number of Defective Inspection Observations }}{\text { Total Number of Inspection Observations }} \times 1,000,000
$$

The Number of Inspection Observation is computed by summing the number of parts inspected on each key. This assumes that each key represents one inspection observation. A variation that is addressed in the DPMO example below is to count more than one observation per key inspected per part. 
The equation for PD is

$$
P D=100 \times \frac{\text { Total Number of Parts Defective }}{\text { Total Number of Parts Inspected (Largest Sample Size) }}
$$

To compute the totals the maximums are taken across the three keys. Thus, in example A,

$$
P D=100 \times \frac{\max (15,1,0)}{\max (15,45,50)}=100 \times \frac{15}{50}=30 \text { percent }
$$

The Total Defective is the line that corresponds to the number of observations found defective, and Total Observations is the line for the number of observations inspected. Consider examples $A$ and $E$. Both fail the $(15,0)$ sampling plan with 15 defectives found. The disposition is to screen the remainder and scrap the nonconforming units. The metric $P D$ as computed for $A$ is $P D=100^{\star 15 / 50}=30$ percent and for $E, P D=$ $100^{\star} 80 / 1000=8.0$ percent. The raw data indicates that the product is nearly 100 percent defective on key X001. PD drastically underestimates the fraction defective in both cases because it mixes the number defective and the number inspected. The 100 percent key provides a larger denomination and the sampling plan limits the number of defectives in the numerator. Note also that none of the information from key X001 is used in PD for example $E$. Had key $X 003$ been better, the contrast would have been worse.

The calculations for PPA, PATF, and DPMO are straightforward. The comparison of PPA to PATF gives a clear indication of the quality of the shipment. The shipment is such that about 20 percent of the parts in the shipment are acceptable, but none of the shipment is acceptable without additional cost and effort. The DPMO calculation reflects the screening of the shipment versus the sample plan for key X002. The small shipment size (A) allows the sampled key to have weight about equal to the screened and 100 percent key. The large shipment size (E) reduces the weight of the sampled key in the DPMO calculation. In these two examples, DPMO went from 283,000 DPMO in A to 430,000 DPMO in E.

The examples $B$ and $F$ have similar sampling results but the disposition is quite different. On the basis of the high defect rate in the key X001 sample, the shipment was returned to the supplier. The calculation of PD and PATF are unchanged. PPA is reduced to zero by the RTV disposition. The DPMO values show a large decrease because of the decision to RTV rather than screen. This decision kept defectives out of the numerator even though it also reduced the denominator (number of observations). This reduction, especially for the large shipment, provides an overly optimistic value for DPMO.

Examples $C, D, G$, and $H$ illustrate conditions that can occur when defective rates are moderate to low. In example $C$ the shipment passes the two sampling keys. All the metrics calculations yield different numbers but similar conclusions. There is some small 
level of defectiveness, but a large portion, if not all, of the product received will be acceptable.

In Example $G$ the sampling plans both fail and the shipment is screened on both keys. Because only eight units were not accepted, PATF has a small positive value $(0.7 \%)$ rather th an zero. PATF usually has a value of zero when the shipment is screened.

Examp!a $D$ is another situation where the shipment is screened but PATF is non-zero. Again the disposition of defective parts is the important factor. Five units were not accepied, but only 35 units were actually screened. Thus, under the current calculation rules ten units were accepted trouble free.

Example $\mathrm{H}$ exhibits moderate defectiveness on all three keys. Both sampling keys fail and the shipment screened. In this case, the nature of keys X001 and X002 are assumed so that the screen was done twice, rather than once on two characteristics. This occurs when the keys are different, such as a marking key inspected on a visual basis and a functional key inspected with electrical test equipment or a gage. In this situation, the parts must be handled twice. Note that the total number screened reflects this.

The four metrics provide values that look somewhat inconsistent but generally distinguish consistently among shipments. All four metrics tend to agree that examples A, B, E, and $F$ are the worst four shipments. PD and DPMO rank $D$ below $F$. The remainder are ranked consistently with this conclusion. The best shipments are not as consistently disting : ished. PATF ranks $C$ as the best shipment while the other three pick G. PATF picks $C$ because the sampling plans sassed and no screening was required. PD, PPA, and PATF give example $G$ a low defectiveness rate including the screening results because none of the three directly depends on whether the shipment is screened.

The examples in this appendix illustrate some of the typical inspection results and corresponding metrics calculations that occur on purchased product. All four metrics discussed converge to the desired target as the defectiveness declines. The metrics are affected by differences in shipment sizes, differences in sample sizes, the final disposition of the part in a shipment, as well as the defectiveness level. 


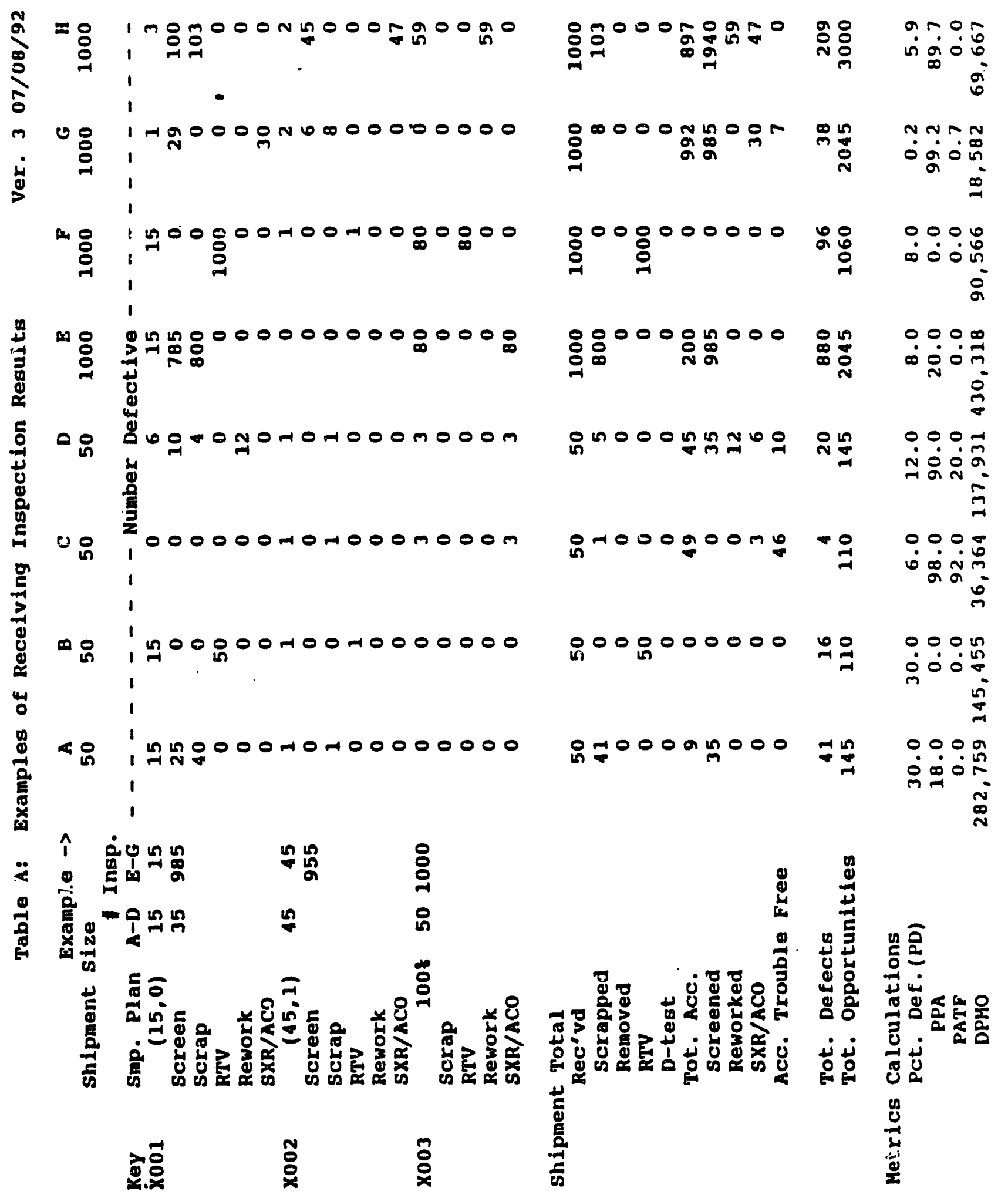


Appendix B

DPMO for an Electronics Assembly 
The example in this section is a logic assembly. The example compares two methods of counting observations for the DPMO metric. From this example, counting according to guidance in DoD-STD-2000 provides a meaningful basis for DPMO.

The Inspection Instruction (II) contains three operations or groups and a total of nine characteristics or keys. A representative II is listed in the Logic Assembly Quality Metric $A$ and contains the three groups. In group 010 there is one key, Key 010 Workmanship. Group 020 has seven keys, 010-070, and group 030 has one key, Key 010 Component Parameters. Under the current DPMO implementation using QARS, the number of observations would be counted as the total number of keys. This total is nine keys.

The groups and keys are also listed along with the possible features, either in terms of defect types or places to check, that are to be observed for the key. For example, group 010, key 010 has seven specific conditions to check plus an "Other." In this example, each condition is counted as an inspection observation. The number of observations for this first key is eight for each part inspected at that group. For key 010 in the 020 group. there are ten screws to be checked. The count for this key is 4 keys $\times 10$ screws $=40$ observations. In like manner, the number of observations is obtained for the other keys. For this rather simple electronic part, the count of observations for each part that is inspected at all three groups is 11,844 .

The same II is listed in Logic Assembly Quality Metric B. Observations are counted by counting the number of components or features that is to be inspected for that key, but the possible defect types are ignored. Thus, group 010 key 010 counts only one observation per part and group 020 key 030 Capacitor Soldering (101 chip capacitors) counts 202 observations (two leads soldered per capacitor). The total count of observations by this method for each part that is inspected at all three groups is 1271. This method of counting observations is based on guidance contained in Dod-STD-2000-1B, Appendix C (dated 15 January 1987). An excerpt illustrating the method is contained in Figure C-3 from that appendix and is included following the Metric B summary page.

The next page, Logic Subassembly DPMO, contains some typical inspection results for 41 parts. The computations are completed assuming that 70 defects were detected during inspection. For the first method, the value is $D P M O(A)=144$. For the second, the value is $D P M O(B)=1343$. If it is assumed that only seven part-key combinations were defective, the QARS method would yield a value of DPMO $=7 /\left(9^{\star} 41\right)^{\star} 1,000,000=18,970$.

Method A provides a much smaller number than Method B and, in general, so will any counting scheme that increases the denominator without at least allowing the numerator to increase by counting more defects. The QARS method yields a much larger number than the other two due to the fact that multiple defects are not counted on individual keys. QARS does collect data on the number of defects. This would allow the counting to be more like the DPMO(B) situation. Also note that Method A would never allow DPMO to be $1,000,000$ (when everything is defective) because defect types such as "excess solder" and "insufficient solder" cannot both be present on a single feature. Method B provides a more meaningful scale according to the definition of a metric. 


\section{Logic Assembly Quality Metric A}

11 Operation 010

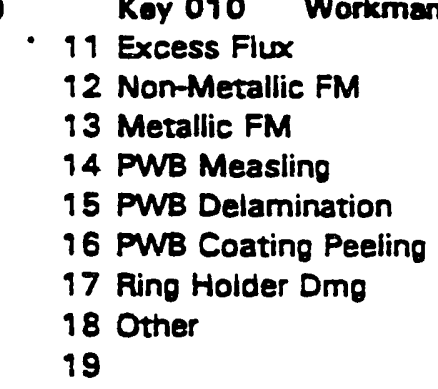

3) Operation 020

4) Oparation 020

5) Operation 020
Koy 020 Logic Ring Soldering

29 Insufficient Solder

22 Excess Solder

23 Excess Voids

24 No Solder

Koy 030 Capacitor Soldering

31 Insufficient Solder

32 Excess Solder

33 Excess Voids

34 No Solder

35 Cracked Solder

36 Cold Solder

37 Disturbed Solder

38 Contaminated Solder

39 Orientation/Location

40 Other

Koy 040 Inductor Soldering

41 Insufficient Solder

42 Excess Solder

43 Excess Voids

44 No Solder

45 Cracked Solder

46 Cold Solder

47 Disturbed Solder

48 Contaminated Solder

49 Orientation/Location

50 Other
1 Ass'y

Observations $=8$

10 Serows

Observations $=40$

1 Ring

Observations $=4$

101 Chip Capacitors

Observations $=2020$

13 inductors

Observations $=260$ 
52 Excess Solder

53 Excess Voids

54 No Solder

55 Cracked Solder

56 Cold Solder

57 Disturbed Solder

58 Contaminated Solder

- 59 Orientation/Location

60 Other

Observations $=4260$

7) Oparation 020

Key 060 Transistor Soldering

61 Insufficient Solder

62 Excess Solder

63 Excess Voids

64 No Solder

65 Cracked Solder

66 Cold Solder

67 Disturbed Solder

68 Comtaminated Solder

69 Orientation/Location

70 Other

Observations $=1940$

8) Oparation 020

Kay 70 IC Soldering

71 Insufficient Solder

72 Excess Solder

73 Excess Voids

74 No Solder

75 Cracked Solder

76 Cold Solder

77 Disturbed Solder

78 Contaminated Solder

79 Orientation/Location

80 Other

38 Transistors

2 IC's, 112 loods

Observations $=1120$

9) Operation 030

Key 010 Component Parameters

11 Marking lllegible

12 Marking Incorrect

13 Marking Missing

14 Missing

15 Crackéd

16 Chipped

17 Damaged

18 Other

Observations $=3032$

Total Observations $=11844$ 


\section{Logic Subassembly Qualilty Metric B}

Note: Based upon DOD 2000 method of determined defect rate.

1) Operation 010

Key 010

19 Excess Flux

12 Non-Metallic FM

13 Metallic FM

14 PWB Measling

15 PWB Delamination

16 PWB Coating Peeling

17 Ring Holder Dmg

18 Other

19

2) Operation 020

$$
\text { Kay } 010
$$

Serow Soldering

11 Insufficient Solder

12 Excess Solder

13 Excess Voids

14 No Solder

3) Operation 020

Key $020 \quad$ Logic Ring Soldering

21 Insutficient Solder

22 Excess Solder

23 Excess Voids

24 No Solder

4) Operation 020

5) Operation 020

Key 030 Capactor Soldering

31 Insutficient Solder

32 Excess Solder

33 Excess Voids

34 No Solder

35 Cracked Solder

36 Cold Solder

37 Disturbed Solder

38 Contaminated Solder

39 Orientation/Location

40 Other

Koy 040 Inductor Soldering

41 Insufficient Solder

42 Excess Solder

43 Excess Voids

44 No Solder

45 Cracked Solder

46 Cold Solder

47 Disturbed Solder

48 Contaminated Solder

49 Orientation/Location

50 Other

Koy 050 Resistor Soldering

51 Insufficient Soider

52 Excess Solder
Observations $=10$

1 Ass'y

Obsarvetions $=1$

10 Seraws

1 Ring -

Observations $=1$

101 Chip Capacitors

Observations $=202$

13 Inductors

Observations $=26$

213 Resistors 
53 Excess Voir's

54 No Solder

55 Cracked Solder

56 Cold Solder

57 Disturbed Solder

58 Contaminated Solder

59 Orientation/Location

60 Other

Observations $=426$

7) Operation 020

Key 060 Transistor Soldering

61 Insufficient Solder

62 Excess Solder

63 Excess Voids

64 No Solder

65 Cracked Solder

66 Cold Solder

67 Disturbed Solder

68 Contaminated Solder

69 Orientation/Location

70 Other

Observations $=114$

8) Operation 020

Koy 70 IC Soldering

71 Insufficient Solder

72 Excess Solder

73 Excess Voids

74 No Solder

75 Cracked Solder

76 Cold Solder

77 Disturbed Solder

78 Contaminated Solder

79 Orientation/Location

80 Other

Observations $=112$

9) Operation 030

Key 010 Component Parameters

11 Marking lllegible

12 Marking Incorrect

13 Marking Missing

14 Missing

15 Cracked

16 Chipped

17 Damaged

18 Other

Observations $=379$

Total Observations $=1271$ 
Logic Subassembly Qualilty Metric B

Note: Based upon DOD 2000 method of determined defect rate.

1) Operation 010

$$
\text { Key } 010
$$

Workmanship

2) Operation 020

3) Operation 020

4) Operation 020

5) Operation 020

6) Operation 020

7) Operation 020

8) Operation 020

9) Operation 030
Key 060

Transistor Soldering

Key 70

IC Soldering

Ker 010

Component Parameters
1 Ass'y

Observations $=1$

10 Screws

Observations $=10$

1 . Fing

Observations $=1$

101 Chip Capacitors

Observations $=202$

13 Inductors

Observations $=26$

213 Resistors

Observations $=426$

38 Transistors

Observations $=914$

2 IC's, 112 leads

Observations $=112$

379 Components

Observatioris $=379$

Total Observations $=1271$ 
DOD-STD-2000-1B

15 JANUARY 1987

APPENDIX C

FIGURE C-3. Calculation of assembly defect rate (see 5.4.4.1.4).

Components

Component Leads

Terminals

Wires

PWB

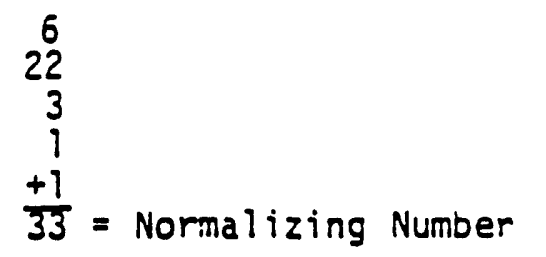

Number of Assembly Defects observed = Assembly Normalizing Number Defect Rate

Sample: If there were 3 Assy Defects fuund on PWB below then $\frac{3}{33}=9.1 \%$ Assembly Defect Rate

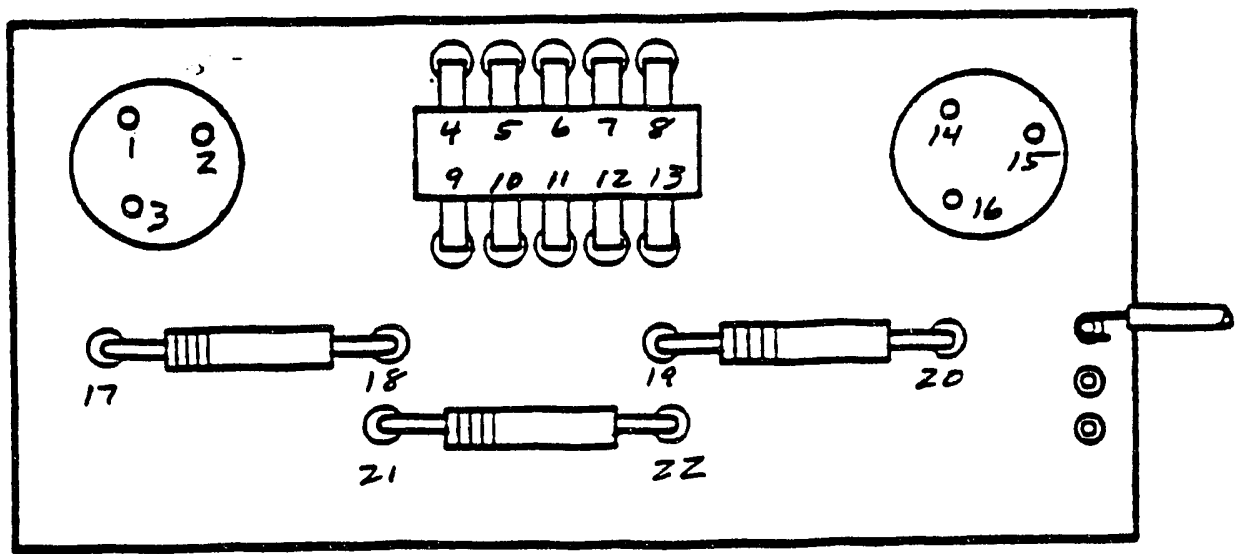




\section{Logic Subassembly DPMO}

$\begin{array}{cc}\text { Components } & \\ \text { PWB } & 1 \\ \text { Scrows } & 10 \\ \text { Logic Rino } & 1 \\ \text { Chip Capacitors } & 101 \\ \text { Chip Inductors } & 13 \\ \text { Transistors } & 38 \\ \text { Chip Resistors } & 213 \\ \text { IC's } & 2 \\ \text { Total } & 379\end{array}$

Yield

$\begin{array}{ll}1 \text { st Visual } & 85 \% \\ \text { 2nd Vis } & 53 \% \\ \text { Ave Yield } & 68 \%\end{array}$

DPMO

Method A (Categories are applied to each solder joint and component to form an observation)

Method B (Each component plus solder joints are observations)

$\operatorname{DPMO}(A)=(70$ Defects $/ 41 * 11,844$ obs. $) * 1,000,000=144$

DPMO $(B)=70 /(41+1271)+1,000,000=1343$ 


\section{Appendix C}

DPMO Example in Mechanical Purchased Products 
An experiment was conducted to evaluate the impact on purchased mechanical products of using a new approach of counting inspection opportunities for the DPMO calculation. Three products were selected that had been received and irspected through receiving inspection.

The lls for these products were reviewed key by key to assess the number of observations actually being made within the normal inspection process. Using the sample plan prescribed by the key and the lot size, the quantity inspected for each key was determined. By multiplying the actual number of observations by the sample size for each key, then adding all the keys, the total number of opfortunities was determined.

The present practice of counting observations is based on one per key, regardless of how many features are referenced in that key. Therefore, the total number of opportunities is calculated by adding the sample size for each key into a total.

The following table reflects a comparison of the results received from computing DPMO by these different methods. Actual reject history is used from the inspection results for the products.

\begin{tabular}{|lcccccc|}
\hline & & & \multicolumn{3}{c}{ Present } & \multicolumn{2}{c|}{ Proposed } \\
\cline { 5 - 7 } & Sot & $\begin{array}{l}\text { Reported } \\
\text { Sefects }\end{array}$ & $\begin{array}{l}\text { Total } \\
\text { Spportunities }\end{array}$ & DPMO & $\begin{array}{l}\text { Total } \\
\text { Opportunities }\end{array}$ & DPMO \\
\hline $\begin{array}{l}\text { Stud } \\
\begin{array}{l}\text { Support } \\
\text { Ring }\end{array}\end{array}$ & 50 & 31 & 432 & 71759 & 758 & 40987 \\
$\begin{array}{l}\text { Outer } \\
\text { Plate }\end{array}$ & 9 & 4 & 135 & 29269 & 639 & 6259 \\
\hline
\end{tabular}

These parts represent a range of complexity. The results indicate that products with multiple features have significantly lower DPMO numbers than those without multiple features. The above examples illustrate this point. The support ring has two separate eight-hole patterns and a multiple-notch pattern. The outer plate has a 32-hole pattern, a 12-hole pattern, and a four-hole pattern. These multiple features compound even further when both size and location of each hole are counted as different observations.

The workmanship key was expanded to two observations for these examples: presence of burrs and surface finish/condition. One observation for all the different attributes of acceptable workmanship does not seem reasonable because many different processes are required. 
In summary, this computation of the DPMO metric is not reflecting all the opportunities that are being observed by normal inspection activities. By simply changing the way in which opportunities are calculated, a significant decrease in absolute DPMO figures will result and at the same time provide a more detailed quality history database, a database that could be more responsive to efforts to identify product drivers and out-of-control processes. 

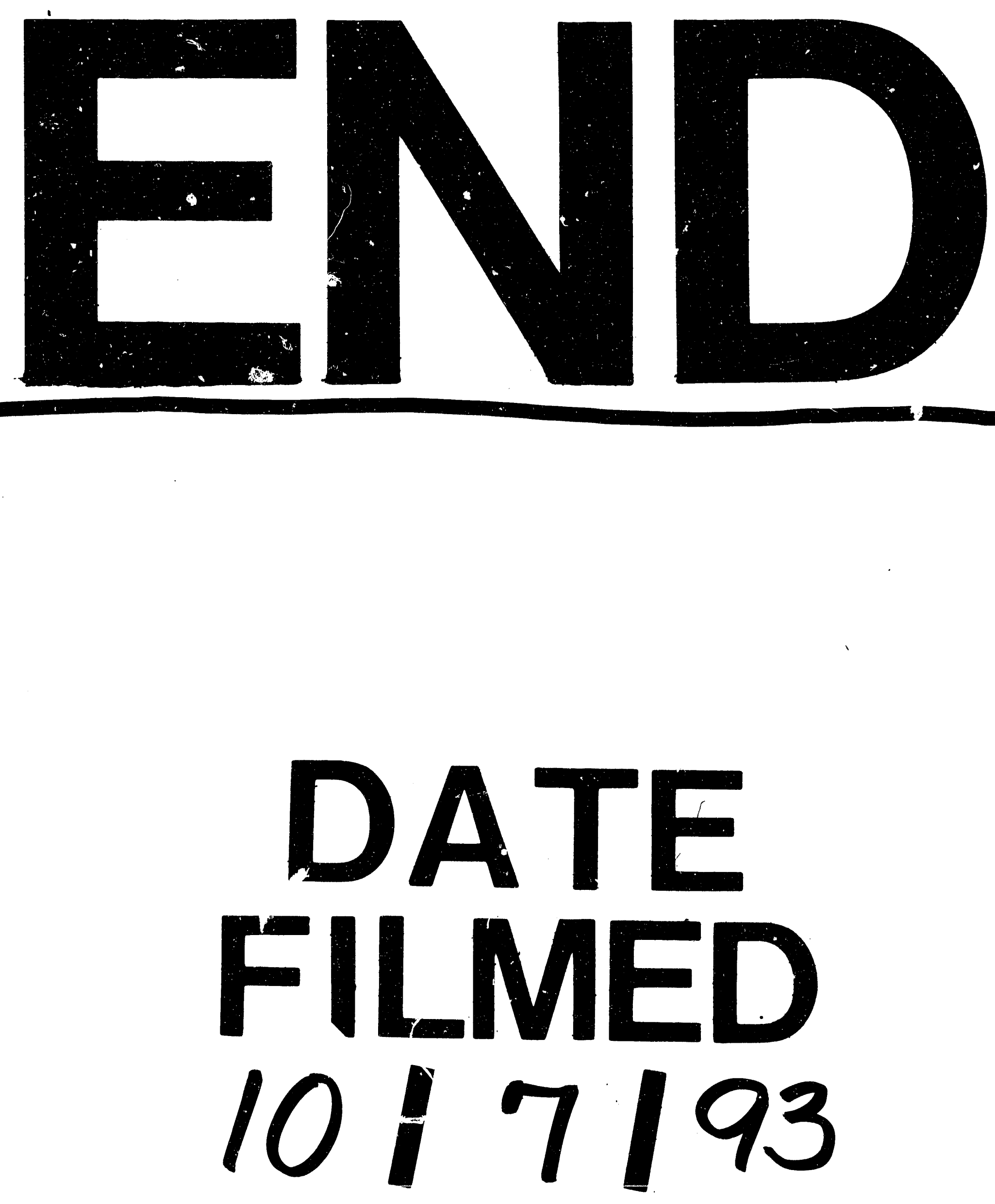

I 
